TITLE:

\title{
Simultaneous detection of vertical and lateral forces by bimodal AFM utilizing quartz tuning fork sensor with a long tip
}

\section{$\operatorname{AUTHOR}(\mathrm{S}):$}

Yamada, Yuya; Ichii, Takashi; Utsunomiya, Toru; Sugimura, Hiroyuki

\section{CITATION:}

Yamada, Yuya ... [et al]. Simultaneous detection of vertical and lateral forces by bimodal AFM utilizing quartz tuning fork sensor with a long tip. Japanese Journal of Applied Physics 2019, 58(9): 095003.

ISSUE DATE:

2019-09-01

URL:

http://hdl.handle.net/2433/244293

\section{RIGHT:}

This is a peer-reviewed, un-copyedited version of an article accepted for publication/published in Japanese Journal of Applied Physics. IOP Publishing Ltd is not responsible for any errors or omissions in this version of the manuscript or any version derived from it. The Version of Record is available online at https://doi.org/10.7567/1347-4065/ab3617.; The full-text file will be made open to the public on 13 August 2020 in accordance with publisher's 'Terms and Conditions for Self-Archiving'.; この論文は出版社版でありません。引用の際には出版社版をご確認ご利用ください。;This is not the published version. Please cite only the published version. 


\title{
Simultaneous detection of vertical and lateral forces by bimodal AFM utilizing quartz tuning fork sensor with a long tip
}

Yuya Yamada, Takashi Ichii*, Toru Utsunomiya and Hiroyuki Sugimura

Department of Materials Science and Engineering, Graduate School of Engineering, Kyoto University, Kyoto 606-8501, Japan

E-mail: ichii.takashi.2m@kyoto-u.ac.jp

\begin{abstract}
Simultaneous detection of vertical and lateral forces at nanoscale by atomic force microscopy (AFM) provides important knowledge in nanotribology. Although silicon (Si) cantilevers are capable of detecting both the forces, it has not been achieved by quartz tuning fork (QTF) sensors including qPlus sensors. In this study, we found that the tip apex of the qPlus sensor with a long tip oscillates vertically at the lowest resonance frequency $\left(f_{1}\right)$ and laterally at the second lowest resonance frequency $\left(f_{2}\right)$ by the finite element method (FEM) simulation. The lateral oscillation was experimentally confirmed by atomic resolution imaging, where the imaged atoms were apparently connected as increasing the oscillating amplitude at $f_{2}$. We also demonstrated the nanometer-scale friction force measurement by using the developed bimodal AFM. The obtained result was in good agreement with the contact-mode lateral force microscopy utilizing a Si cantilever.
\end{abstract}

\section{Key words}

multifrequency atomic force microscopy, qPlus sensor, atomic resolution, nanotribology 


\section{Introduction}

Atomic force microscopy $(\mathrm{AFM})^{1)}$ is a powerful tool for structural analysis of solid surfaces or solid/liquid interfaces. It enables nanoscale imaging using force acting between a tip and surface. The silicon ( $\mathrm{Si}$ ) cantilevers utilizing an optical beam deflection technique are most widely used as the force sensor, where both the vertical deflection and lateral torsion of the cantilever are detected by a four-segment photodiode. ${ }^{2)}$ The vertical force is usually used for topographic imaging. In contrast, the lateral force reflects a friction force acting between the tip and surface. ${ }^{3)}$ Nanoscale and atomic scale analysis of friction is of crucial importance for nanotribology. ${ }^{4,5)}$ Recently, S. Kawai and coworkers demonstrated vertical and lateral bimodal AFM, which uses lowest flexural and torsional resonance modes of a Si cantilever, in an ultrahigh vacuum (UHV) environment. ${ }^{6-8)}$ They achieved simultaneous detection of the vertical force and the atomically resolved lateral force.

In addition to Si cantilevers, quartz tuning fork (QTF) sensors, especially qPlus sensors, ${ }^{9)}$ are also widely used as the AFM force sensor. The qPlus sensor with a tip attached perpendicularly to the QTF beam has exhibited atomic resolution in various environments, such as UHV, ${ }^{10)}$ ambient air ${ }^{11,12)}$ and liquids. ${ }^{13,14)}$ The qPlus sensor can also detect the lateral force by attaching a tip parallel to the long axis of the QTF beam. ${ }^{15-17)}$ However, simultaneous detection of vertical and lateral forces by a qPlus sensor is difficult because torsional deformation of QTF is undetectable by its piezoelectric effect. Therefore, simultaneous excitation of flexural and torsional modes similar to Si cantilevers is useless for qPlus sensors. To the best of our knowledge, the only one report of atomic resolution bimodal AFM by a qPlus sensor has been published, where the tip apex oscillates in the vertical direction at both the two resonance frequencies. ${ }^{18)}$ A different design of a quartz sensor, that uses first two flexural modes of the quartz beam supported at both ends and a tip is attached at center of the beam, was proposed to detect vertical and lateral forces simultaneously. ${ }^{19)}$ However, no experiments has been demonstrated with this sensor. A short tip with a small mass is widely used for QTF sensors because a mass of the tip decreases the resonance frequency of the QTF, which generally suppresses the force sensitivity and complicates the motion of QTF.

In this report, the qPlus sensor with a long tip $(>1 \mathrm{~mm})$ attached perpendicularly to the long axis of the QTF beam was adopted. It was found that the tip apex of the sensor oscillates vertically at the lowest resonance frequency, $f_{1}$, and laterally at the second lowest resonance frequency, $f_{2}$, by the finite element method (FEM) simulation. We demonstrated atomic resolution imaging of $\operatorname{KBr}(100)$ and nanometer-scale friction force measurements on 
graphene oxide (GO) sheets spincoated on a highly oriented pyrolytic graphite (HOPG) substrate by the bimodal AFM using $f_{1}$ and $f_{2}$ of the qPlus sensor. In the atomic resolution images, the atoms of a $\mathrm{KBr}(100)$ surface were apparently connected when the oscillating amplitude at $f_{2}$ increased. This indicates that the tip apex oscillates laterally at $f_{2}$. In the bimodal AFM results of the GO-coated HOPG substrate, the GO-covered area showed larger energy dissipation compared to the bare HOPG surface at $f_{2}$, which is in good agreement with contact-mode lateral force microscopy (LFM) utilizing a Si cantilever. These simulation and experimental results revealed that the lateral friction force is simultaneously detectable with the vertical force by a qPlus sensor with a long tip.

\section{Experimental methods}

Figure 1 shows a photograph of a qPlus sensor used in this study. One prong of a commercial quartz tuning fork (QTF, STATEK Co., TFW 1165, spring constant $1884 \mathrm{~N} \mathrm{~m}^{-}$ ${ }^{1}$ ) was bound to a mount. A tungsten wire (The Nilaco Co., diameter $0.1 \mathrm{~mm}$ ) was electrochemically etched in potassium hydroxide aqueous solution $\left(1.2 \mathrm{~mol} \mathrm{~L}^{-1}\right)$ and glued to the other prong by EPO-TEK H70E (Epoxy Technology, Inc.). The length of the tungsten tip was 1.2-1.5 mm, which is relatively larger than the typical value used in qPlus sensors.

FEM simulation was carried out by FreeCAD $0.17,{ }^{20)}$ an open source $3 \mathrm{D}$ parametric modeler with FEM solvers, in order to reveal the oscillation modes of the qPlus sensor numerically. Table I shows physical parameters of the QTF and the tungsten tip. It was approximated that the QTF and the tip were bonded by a plane and the QTF except for the prong with the tip was fixed. Creation of a mesh and solution of resonance modes were conducted by Gmsh and Calculix on FreeCAD.

The experiment was performed by a system based on a commercial AFM (JEOL, JSPM5200) of which original AFM head was replaced by a home-built AFM head made for a qPlus sensor. Figure 2 shows a block diagram of the bimodal AFM setup. The sensor was mechanically oscillated by lead zirconate titanate (PZT) piezoelectric plate driven at two different resonance frequencies simultaneously. The deflection signal of the sensor was amplified by a differential current amplifier ${ }^{21)}$ embedded in the AFM head. Frequency shift of the lowest resonance frequency $\left(\Delta f_{1}\right)$ was detected by a commercial FM demodulator (Kyoto Instruments, KI-2001) with some modifications, and amplitude and phase shift at the second lowest resonance frequency $\left(A_{2}, \varphi_{2}\right)$ were detected by lock-in amplifier (NF Electronic Instruments, LI 5640). Topographic images were obtained as two-dimensional maps of the tip trajectory where $\Delta f_{1}$ was kept constant, which is so-called frequency 
modulation (FM) mode.

A $\operatorname{KBr}(100)$ substrate was used as a standard sample for atomic resolution imaging. 1-

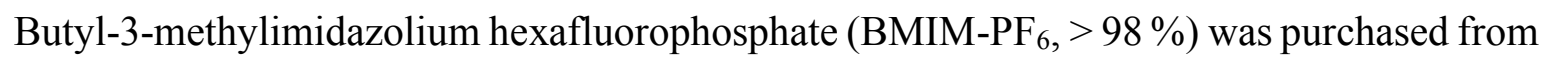
Tokyo Chemical Industry Co., Ltd. and used without further purification. The saturated $\mathrm{KBr}$ solution of BMIM-PF 6 was prepared by adding $1 \mathrm{wt} \%$ of $\mathrm{KBr}$ powder (Nacalai Tesque Inc., $>99 \%$ ) and agitating on a tube rotator for 1 day. After that, (100) oriented $\mathrm{KBr}$ single crystal (Furuuchi Chemical Co.) was cleaved by using sharp knife and immediately supernatant of the solution $(0.5 \mu \mathrm{L})$ was dropped on the cleaved $\operatorname{KBr}(100)$ surface. This sample was left in the dry chamber (dew point $<-50^{\circ} \mathrm{C}$ ) for 1 day, and then it was investigated by the bimodal AFM with the long tip qPlus sensor.

$\mathrm{GO}$ and graphite were used as a test sample for friction force measurements in ionic liquid. GO sheets were prepared through a modified Hummers' method. ${ }^{22)}$ A highly oriented pyrolytic graphite (HOPG, SPI supplies, SPI-2 grade, $10 \times 10 \times 2 \mathrm{~mm}$ ) was cleaved by using scotch tape, and then the GO 1-propanol dispersion was spincoated onto the cleaved HOPG surface. 1-Ethyl-3-methylimidazolium bis(trifluoromethylsulfonyl)imide (EMIM-Tf $2 \mathrm{~N}$, purchased from Kanto Chemical Co., Inc.) $0.5 \mu \mathrm{L}$ was dropped on the substrate, and then the sample was investigated by the bimodal AFM using another qPlus sensor.

\section{Results and discussion}

Figure 3 shows thermal Brownian spectra of the qPlus sensor obtained in ambient air. The frequencies of the first two resonance modes were $f_{1}=13.509 \mathrm{kHz}$ and $f_{2}=160.760 \mathrm{kHz}$. The quality factors (Q factors) of $f_{1}$ and $f_{2}$ were 2439 and 423 respectively. These two resonance modes were adopted to this bimodal AFM experiment. The 2nd resonance frequency, $f_{2}$, was 11.9 times higher than $f_{1}$. It is quite different from theoretical values of cantilever's 2 nd and 3rd resonance frequencies (6.27 times and 17.6 times of $f_{1}$ ), because higher resonance modes of qPlus sensors are drastically affected by mass and rotation inertia of the long tip. ${ }^{23)}$ Considering that, resonance frequencies and modes were calculated by the method in Ref. 23. In this method only in-plane oscillations (vertical oscillations) of the QTF were considered. The tip was approximated as a rigid circular column length of $1.17 \mathrm{~mm}$ in order to equalize its mass to the actual amount. The solutions of first three resonance frequencies were $13.8 \mathrm{kHz}, 64.2 \mathrm{kHz}$ and $240 \mathrm{kHz}$, and the oscillation modes of them are shown in Fig. 4. That is in good agreement with the experimental values of $f_{1}$ but not $f_{2}$, which suggests the possibility that the tip doesn't behave as a rigid body at $f_{2}$. In fact, the spring constant of the tip is $2730 \mathrm{~N} \mathrm{~m}^{-1}$ (calculated by approximating the tip as a circular 
column), which is the same in the order of magnitude as that of the QTF beam in the inplane direction, $1884 \mathrm{~N} \mathrm{~m}^{-1}$. Furthermore, the spring constants of higher resonance modes are generally much higher than that of the fundamental mode. Therefore, the rigid-tip approximation was not suitable in this case. The results also suggested the presence of outof-plane or torsional oscillation modes. These modes, however, are considered less likely to be detected by piezoelectric effect of QTFs because they are manufactured for sensing inplane oscillation.

The calculated frequencies at higher resonance modes under the rigid-tip approximation are obviously different from the experimental values. In order to investigate resonance modes of the qPlus sensor more exactly, FEM simulation was carried out by FreeCAD. The tip attached to the qPlus sensor had tilted $8^{\circ}$ from the perpendicular direction of the QTF beam (Fig. 5 (a)), which was reflected in the simulation model (Fig. 5 (b)). The simulation results of first six resonance frequencies were obtained at $7.94 \mathrm{kHz}, 13.4 \mathrm{kHz}, 32.6 \mathrm{kHz}$, $43.5 \mathrm{kHz}, 94.0 \mathrm{kHz}$ and $162 \mathrm{kHz}$, and the oscillation modes of them are shown in Fig. 5 (c)(h). The frequencies of the oscillation modes shown in Fig. 5 (d) and (h) agrees well to the experimental values of $f_{1}$ and $f_{2}$ respectively, and the QTF vertically oscillates at both $f_{1}$ and $f_{2}$. Although the simulation suggests that the QTF oscillates in the in-plane direction at $43.5 \mathrm{kHz}$ (Fig. 5 (f)), the deflection signal of this mode was not detected by its piezoelectric effect. This is ascribed that this mode mostly consists of the tip oscillation and the QTF oscillation is negligibly small. The others consist of out-of-plane or torsional oscillation, which were not detectable by the piezoelectric effect. Figure 5 (i) and (j) are side views of resonance modes at $f_{1}$ and $f_{2}$ (Fig. $5(\mathrm{~d})$ and (h)), respectively. According to them, the tip also bends at $f_{2}$ and the tip apex oscillates in a direction orthogonal to that of $f_{1}$. The deflection of the QTF end at $f_{2}$ was relatively small, and the tip bended to $+\mathrm{x}(-\mathrm{x})$ direction when middle part of the QTF deflected to $+\mathrm{z}(-\mathrm{z})$ direction. Figure $5(\mathrm{k})$ is the image of the tip apex displacement at $f_{2}$ viewed from a side. The misalignment from the lateral direction was $7^{\circ}$, which indicates that the crosstalk of vertical force was suppressed to $\sim 12 \%$ and the lateral force was dominantly detected. Note that, we also found that the misalignment can be decreased to $1^{\circ}$ by elongating the tip length to $2 \mathrm{~mm}$, where the crosstalk is suppressed less than $2 \%$. Figure 5 (l) is the image of the 2 nd resonance mode at $f_{2}$ viewed from the bottom. It was found that the angle formed by the direction of the tip apex oscillation and the long axis of the QTF is about $30^{\circ}$. This is probably because the tip was attached on the side of the QTF and that made some torque around the long axis of the QTF. Additionally, we have also confirmed that the effect of the adhesive on the oscillation modes was 
negligibly small. At this stage, it is considered that the tip apex oscillates in the lateral direction at $f_{2}$, while it is difficult to interpret the deflection signal of $f_{2}$ to its actual amplitude because of the complex coupled motion of the QTF and the tip.

In the FEM simulation, the tip apex of qPlus sensor with a long tip oscillates vertically at $f_{1}$ and laterally at $f_{2}$. In order to experimentally confirm this simulation result, atomic resolution imaging was conducted by the bimodal AFM with the qPlus sensor. Figure 6 (a)(d) are atomically resolved $\mathrm{KBr}(100)$ surface images and their Fast Fourier Transform (FFT) patterns obtained with the same fast scan direction in the saturated $\mathrm{KBr}$ solution of BMIM$\mathrm{PF}_{6} . \mathrm{KBr}$ has a rock-salt structure with a lattice constant $a=660 \mathrm{pm}$ (Fig. 6 (e)). Figure 6 (a) was obtained by operating only 1 st mode with the amplitude $A_{1}=180 \mathrm{pm}$. The squarelattice bright spots with the lattice spacing of $475 \mathrm{pm}$ were clearly imaged, which are in good agreement with the spacing between equally charged ions, $\frac{a}{\sqrt{2}}$. Thus, the bright spots represent the periodic sites of only one atomic species as described at previous reports. ${ }^{11,18,24)}$ Figure 6 (b), (c) and (d) were obtained by bimodal AFM operating with constant $A_{1}$ (180 pm) and various $A_{2}(28.3 \mathrm{mV}, 56.5 \mathrm{mV}$ and $113 \mathrm{mV})$. The lateral amplitude $A_{2}$ are described by the detected signal voltage because interpretation from the voltage to the actual lateral amplitude has not been achieved. Although the separated bright spots forming a squarelattice structure were imaged in Fig. 6 (a) and (b), the image changed to stripe-like patterns as $A_{2}$ was increased, which can be also confirmed in the FFT patterns. That is, the imaged atoms were apparently connected to each other. These results are quite different from the previous report in which a short tip was used. ${ }^{18)}$ In atomic resolution images obtained by dynamic LFM (DLFM) ${ }^{25)}$ and scanning tunneling microscopy (STM) combined with DLFM $^{16,26,27)}$ in which the tip apex oscillates laterally, neighboring atoms in the direction of the tip dithering were apparently connected. It should be noted that the AFM images obtained in this experiment are flipped upside down because of our instrumental setting. In order that, the direction of the observed stripes corresponds well to the direction of the tip apex oscillation in the FEM simulation (Fig. 5 (1)). Therefore, these experimental results revealed that the tip apex oscillates laterally at $f_{2}$. In addition, considering the fact that atoms were separately imaged in Fig.6 (b) with $A_{2}=28.3 \mathrm{mV}$ and were apparently connected in (c) with $A_{2}=56.5 \mathrm{mV}$, the actual lateral amplitude of the tip apex at $f_{2}$ can be roughly estimated to be the same order as the lattice spacing.

It was confirmed by the atomic resolution imaging of $\mathrm{KBr}(100)$ that the tip apex of a qPlus sensor with a long tip oscillates laterally at $f_{2}$. The next step is the simultaneous detection of vertical and lateral forces by the bimodal AFM. Figure 7 (a)-(c) show bimodal AFM results 
of the GO-coated HOPG substrate obtained in EMIM-Tf 2 N with another qPlus sensor (tip length $1.68 \mathrm{~mm}, f_{1}=11.222 \mathrm{kHz}, f_{2}=133.46 \mathrm{kHz}$ ). We found that many protrusions were distributed only in the upper area of the topographic image (Fig. 7 (a)). These are typical structures of GO sheets, which are originated from the oxygen functional groups and the defects. $^{28)}$ Thus we can conclude that the upper area and the lower area correspond to the GO-covered area and the bare HOPG surface, respectively. The GO-covered area exhibits larger amplitude attenuation and phase shift of the 2nd mode compared to the HOPG area as shown in Fig. 7 (b) and (c). We also found that the height variation in the bare HOPG did not affect the amplitude and the phase of the 2nd mode, which indicated that the surface properties are detected by the 2 nd mode without topographic effects. In dynamic-mode AFM, the energy dissipated by the tip-sample interaction $\left(E_{\mathrm{dis}}\right)$ is given by $\left.{ }^{29}\right)$

$$
E_{\text {dis }}=\frac{\pi k A^{2} f}{Q}\left(\frac{Q A_{\mathrm{d}}}{A} \sin (-\varphi)-\frac{f}{f_{0}}\right),
$$

where $k, Q, A_{\mathrm{d}}$ and $f_{0}$ are spring constant, quality factor, driving amplitude and natural frequency of the sensor, respectively. Then, $A, \varphi$ and $f$ represent amplitude, phase shift and frequency when the tip approaches the surface. Since $f \simeq f_{0}$ and $Q A_{\mathrm{d}}$ is equal to free amplitude at resonance frequency $\left(A_{0}\right), E_{\mathrm{dis}}$ can be approximated as

$$
E_{\mathrm{dis}}=\frac{\pi k A^{2} f_{0}}{Q}\left(\frac{A_{0}}{A} \sin (-\varphi)-1\right)
$$

Although the absolute value of the energy dissipation cannot be determined because the actual lateral amplitude is unknown, the ratio of the energy dissipation on GO and HOPG can be derived by using the above equation. According to the histograms (Fig. 7 (d), (e)), the averaged amplitude and the averaged phase shift of the 2nd mode on the GO-covered area were $11.43 \mathrm{mV}$ and $-89.27^{\circ}$, and those on the bare HOPG area were $11.68 \mathrm{mV}$ and $89.88^{\circ}$. Substituting them into the above equation, we got $E_{\text {dis GO }}=2.1 E_{\text {dis HOPG }}$. The energy dissipation in the lateral oscillation can be originated from the friction force acting between the tip and the surface by considering the motion equation. Therefore, we concluded that larger friction force was detected on GO sheets than on HOPG by the bimodal AFM. The result was in qualitative agreement with that of a previous report by contact-mode LFM utilizing diamond-like carbon (DLC)-coated Si cantilever, where GO showed larger friction force than HOPG. ${ }^{30)}$ Thus, it is considered that vertical and lateral forces are simultaneously detectable by the bimodal AFM using the qPlus sensor with a long tip.

\section{Conclusion}


We demonstrated simultaneous detection of vertical and lateral forces by the bimodal AFM utilizing a qPlus sensor with a long tip. It was found that the tip apex of the qPlus sensor oscillates laterally at $f_{2}$ by FEM simulation, and it was confirmed by atomic resolution imaging of $\mathrm{KBr}(100)$ surface. Friction force on the GO-coated HOPG substrate in EMIM- $\mathrm{Tf}_{2} \mathrm{~N}$ was detected by the bimodal AFM, and the results agreed well to the contactmode LFM study. It is remarkable that vertical and lateral forces are detectable by the qPlus sensor without special modifications.

This method would be quite useful for nanoscale analysis of lubrication. Structural analysis of lubricant-solid interface has been performed by FM-AFM, and the lubrication layer was visualized. ${ }^{31,32)}$ As a further study, the direct detection of the lateral force at the interface will be achieved by this bimodal AFM. The quantitative detection of lateral force is necessary for these applications. Further research is required for calibration of the actual lateral amplitude, decrease of the misalignment from the lateral direction and quantification of the detected lateral force.

\section{Acknowledgement}

This work was supported by a Grant-in-Aid for Scientific Research B (No. 17H02787) from Japan Society for Promotion of Science (JSPS). 


\section{References}

1) G. Binnig, C.F. Quate, and C. Gerber, Phys. Rev. Lett. 56, (1986)

2) G. Meyer and N.M. Amer, Appl. Phys. Lett. 57, 2089 (1990)

3) C.M. Mate, G.M. McClelland, R. Erlandsson, and S. Chiang, Phys. Rev. Lett. 59, 1942 (1987)

4) B. Bhushan, J.N. Israelachvili, and U. Landman, Nature 374, 607 (1995)

5) G.V. Dedkov, Uspekhi Fiz. Nauk 170, 585 (2000)

6) S. Kawai, F.F. Canova, T. Glatzel, T. Hynninen, E. Meyer, and A.S. Foster, Phys. Rev. Lett. 109, 146101 (2012)

7) S. Kawai, T. Glatzel, S. Koch, B. Such, A. Baratoff, and E. Meyer, Phys. Rev. B 81, $085420(2010)$

8) F. Federici Canova, S. Kawai, C. de Capitani, K. Kan'no, T. Glatzel, B. Such, A.S.

Foster, and E. Meyer, Phys. Rev. Lett. 110, 203203 (2013)

9) F.J. Giessibl, Appl. Phys. Lett. 73, 3956 (1998)

10) F.J. Giessibl, Appl. Phys. Lett. 76, 1470 (2000)

11) D.S. Wastl, A.J. Weymouth, and F.J. Giessibl, Phys. Rev. B 87, 245415 (2013)

12) D.S. Wastl, A.J. Weymouth, and F.J. Giessibl, ACS Nano 8, 5233 (2014)

13) T. Ichii, M. Fujimura, M. Negami, K. Murase, and H. Sugimura, Jpn. J. Appl. Phys.

51, 08KB08 (2012)

14) T. Ichii, M. Negami, and H. Sugimura, J. Phys. Chem. C 118, 26803 (2014)

15) F.J. Giessibl, M. Herz, and J. Mannhart, Proc. Natl. Acad. Sci. 99, 12006 (2002)

16) A.J. Weymouth, D. Meuer, P. Mutombo, T. Wutscher, M. Ondracek, P. Jelinek, and F.J. Giessibl, Phys. Rev. Lett. 111, 126103 (2013)

17) A.J. Weymouth, E. Riegel, S. Matencio, and F.J. Giessibl, Appl. Phys. Lett. 112, 181601 (2018)

18) H. Ooe, D. Kirpal, D.S. Wastl, A.J. Weymouth, T. Arai, and F.J. Giessibl, Appl. Phys. Lett. 109, 141603 (2016)

19) J. Stirling, Beilstein J. Nanotechnol. 4, 370 (2013)

20)"FreeCAD", https://www.freecad.io

21) F. Huber and F.J. Giessibl, Rev. Sci. Instrum. 88, 073702 (2017)

22) M. Hirata, T. Gotou, S. Horiuchi, M. Fujiwara, and M. Ohba, Carbon N. Y. 42, 2929 (2004)

23) R.C. Tung, T. Wutscher, D. Martinez-Martin, R.G. Reifenberger, F. Giessibl, and A. Raman, J. Appl. Phys. 107, 104508 (2010) 
24) S. Kawai, T. Glatzel, S. Koch, B. Such, A. Baratoff, and E. Meyer, Phys. Rev. Lett. 103, 220801 (2009)

25) S. Kawai, S. Kitamura, D. Kobayashi, and H. Kawakatsu, Appl. Phys. Lett. 87, 173105 (2005)

26) S. Kawai, N. Sasaki, and H. Kawakatsu, Phys. Rev. B 79, 195412 (2009)

27) N. Sasaki, S. Kawai, and H. Kawakatsu, Phys. Rev. B 80, 193402 (2009)

28) S. Katano, T. Wei, T. Sasajima, R. Kasama, and Y. Uehara, Phys. Chem. Chem. Phys.

20, 17977 (2018)

29) J.P. Cleveland, B. Anczykowski, A.E. Schmid, and V.B. Elings, Appl. Phys. Lett. 72 , 2613 (1998)

30) D. Berman, A. Erdemir, A. V. Zinovev, and A. V. Sumant, Diam. Relat. Mater. 54, 91 (2015)

31) T. Hirayama, R. Kawamura, K. Fujino, T. Matsuoka, H. Komiya, and H. Onishi, Langmuir 33, 10492 (2017)

32) H. Okubo and S. Sasaki, Tribol. Lett. 67, 3 (2019) 


\section{Figure captions}

Fig. 1. A photograph of a qPlus sensor

Fig. 2. A schematic diagram of the bimodal AFM setup in this work. Frequency shift of 1st mode $\left(\Delta f_{1}\right)$ was detected by the frequency detector, and topographic images were obtained as two-dimensional tip trajectories, where $\Delta f_{1}$ was kept constant. Amplitude and phase shift of 2 nd mode $\left(A_{2}, \varphi_{2}\right)$ were observed by a lock-in amplifier.

Fig. 3. Thermal noise spectra of the qPlus sensor obtained in ambient air (a) around 1st resonance frequency $\left(f_{1}=13.509 \mathrm{kHz}, \mathrm{Q}_{1}=2439\right)(\mathrm{b})$ around 2 nd resonance frequency $\left(f_{2}=160.760 \mathrm{kHz}, \mathrm{Q}_{2}=423\right)$

Fig.4. Calculation results of resonance modes obtained by the method in Ref. 23 approximating the tip as a rigid circular column. The solutions of first three resonance frequencies were $13.8 \mathrm{kHz}$ (red solid line), $64.2 \mathrm{kHz}$ (blue broken line) and $240 \mathrm{kHz}$ (green dashed dotted line).

Fig. 5. (a) A optical microscope image of the tip attached to the qPlus sensor used in this experiment. The tip was tilted $8^{\circ}$ from the long axis of the QTF. (b) A 3D parametric model of the qPlus sensor. Red bodies indicated fixed boundary condition. (c)-(h) FEM simulation results of resonance modes. First six resonance frequencies were $7.94 \mathrm{kHz}(\mathrm{c}$, out-of-plane), $13.4 \mathrm{kHz}$ (d, in-plane), $32.6 \mathrm{kHz}$ (e, torsion), $43.5 \mathrm{kHz}$ (f, in-plane and tipbending), $94.0 \mathrm{kHz}$ (g, out-of-plane and tip-bending) and $162 \mathrm{kHz}$ (h, in-plane and tipbending). (i) The side view of (d). (j) The side view of (h). (k) The tip apex of (h) viewed from a side. The misalignment from lateral direction is $7^{\circ}$. (l) The tip apex of (h) viewed from bottom. The dithering direction of the tip apex tilted $30^{\circ}$ from the long axis of the quartz beam.

Fig. 6. (a)-(d) Topographic images and its FFT patterns of $\mathrm{KBr}(100)$ obtained in $\mathrm{KBr}$ saturated BMIM-PF 6 by the bimodal AFM with constant $A_{1}(180 \mathrm{pm})$ and different $A_{2}$. 
$\Delta f_{1}$ was kept constant during each imaging. (a) $A_{2}=0 \mathrm{mV}, \Delta f_{1}=+250 \mathrm{~Hz}$ (b) $A_{2}=28.3$ $\mathrm{mV}, \Delta f_{1}=+280 \mathrm{~Hz}$ (c) $A_{2}=56.5 \mathrm{mV}, \Delta f_{1}=+290 \mathrm{~Hz}$ (d) $A_{2}=113 \mathrm{mV}, \Delta f_{1}=+260 \mathrm{~Hz}$ (e) A schematic illustration of $\operatorname{KBr}(100)$ surface and the tip dithering direction at $f_{2}$

Fig. 7. The friction measurement result of the GO-coated HOPG substrate obtained in EMIM-Tf 2 N. (a) Topography, (b) 2nd mode amplitude, (c) 2nd mode phase, They were obtained with $A_{1}=190 \mathrm{pm}, A_{2}=11.9 \mathrm{mV}$ and $\Delta f_{1}=+82 \mathrm{~Hz}$. (d) Histogram of (b), (e) Histogram of (c), The red and blue curves are results of fitting with Gaussian functions. 


\section{Table}

Table I. The physical properties of the QTF and the tungsten tip used for the calculation and the FEM simulation.

\begin{tabular}{|c|c|c|c|}
\hline \multicolumn{2}{|c|}{ quartz tuning fork } & \multicolumn{2}{c|}{ tungsten tip } \\
\hline Young's modulus & $80 \mathrm{GPa}$ & Young's modulus & $345 \mathrm{GPa}$ \\
\hline Poisson's ratio & 0.17 & Poisson's ratio & 0.284 \\
\hline density & $2.65 \mathrm{~g} / \mathrm{cm}^{3}$ & density & $19.25 \mathrm{~g} / \mathrm{cm}^{3}$ \\
\hline beam width & $0.213 \mathrm{~mm}$ & length & $1.33 \mathrm{~mm}$ \\
\hline beam length & $2.357 \mathrm{~mm}$ & diameter & $0.1 \mathrm{~mm}$ \\
\hline beam thickness & $0.127 \mathrm{~mm}$ & \multicolumn{2}{|c}{} \\
\cline { 1 - 2 } & \multicolumn{2}{|c}{}
\end{tabular}




\section{Figures}

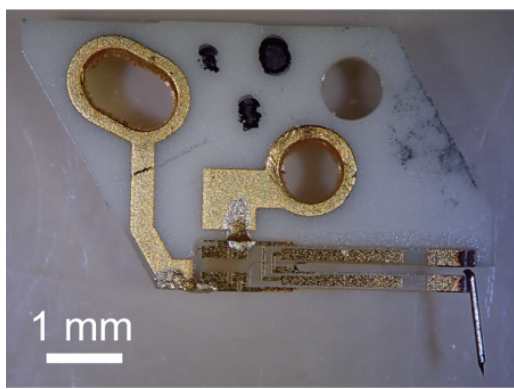

Fig. 1

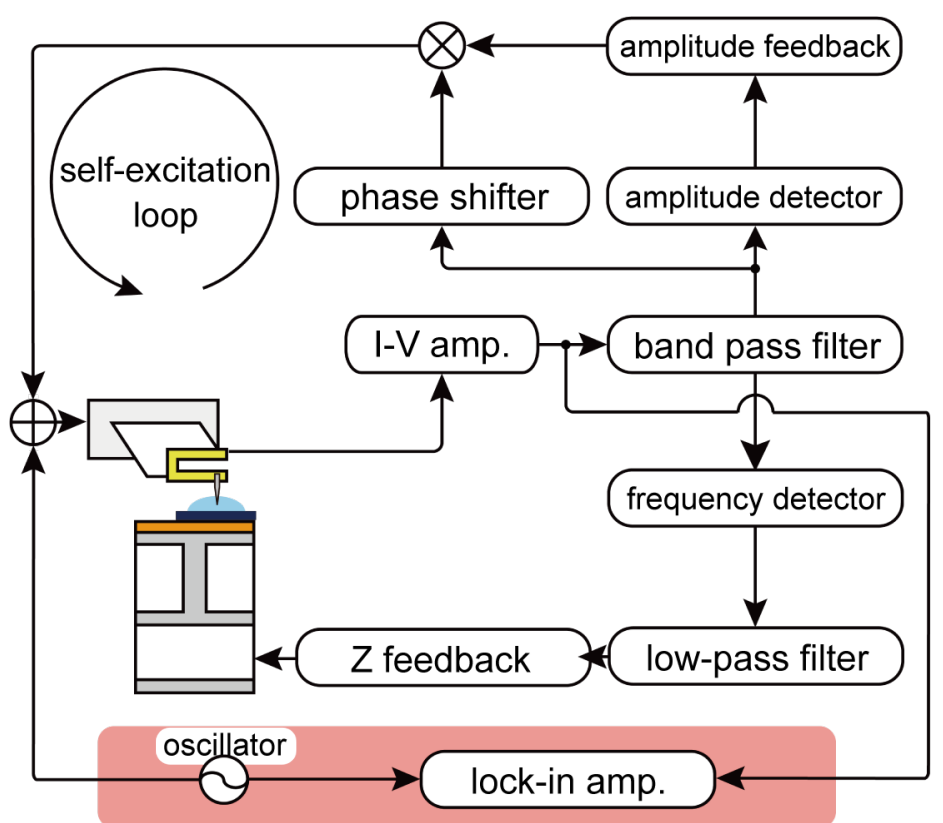

Fig. 2 

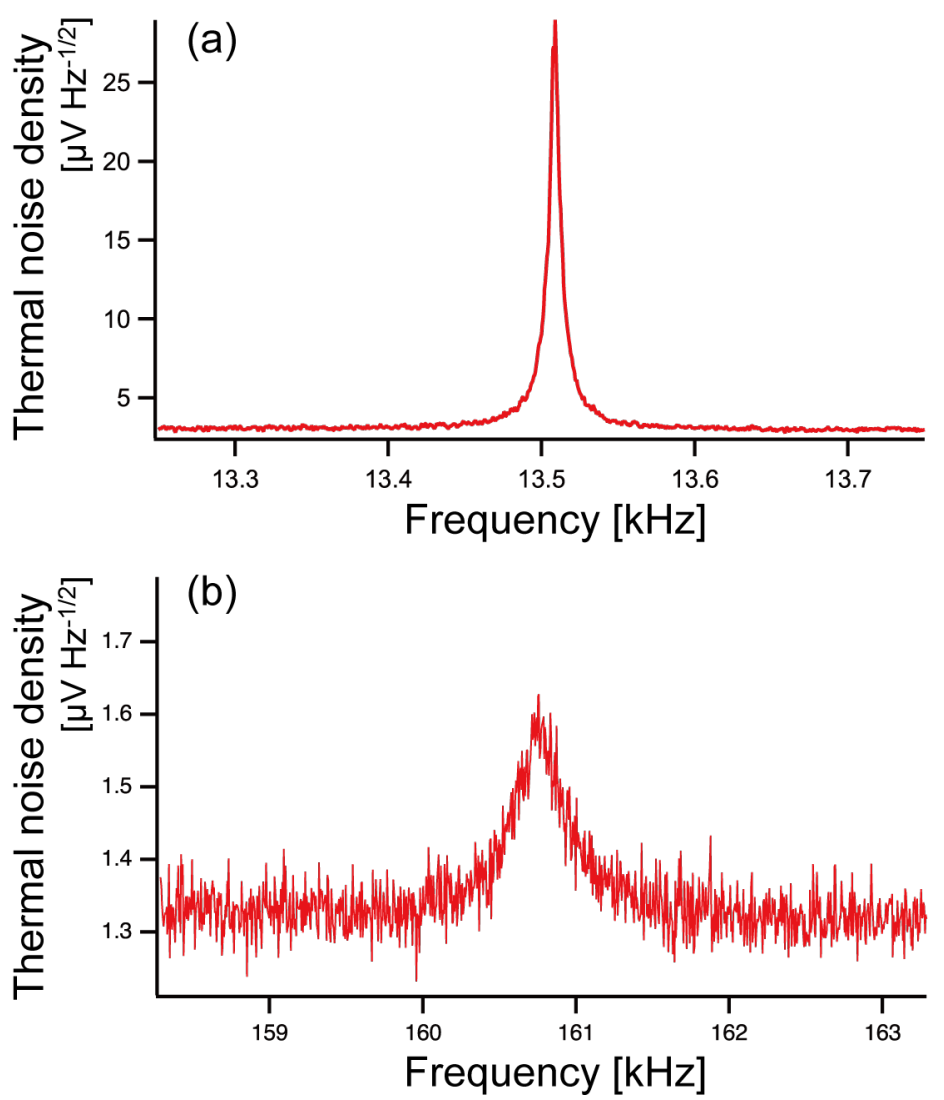

Fig. 3

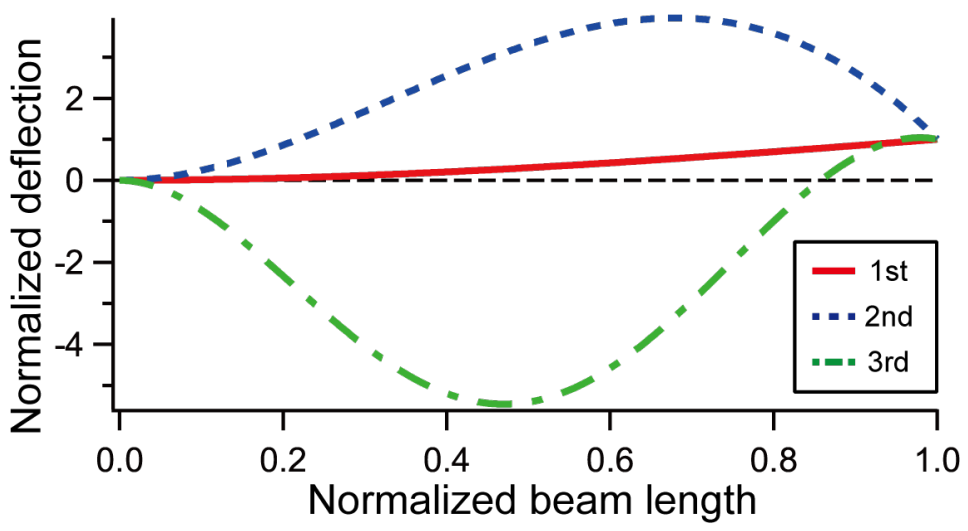

Fig.4 

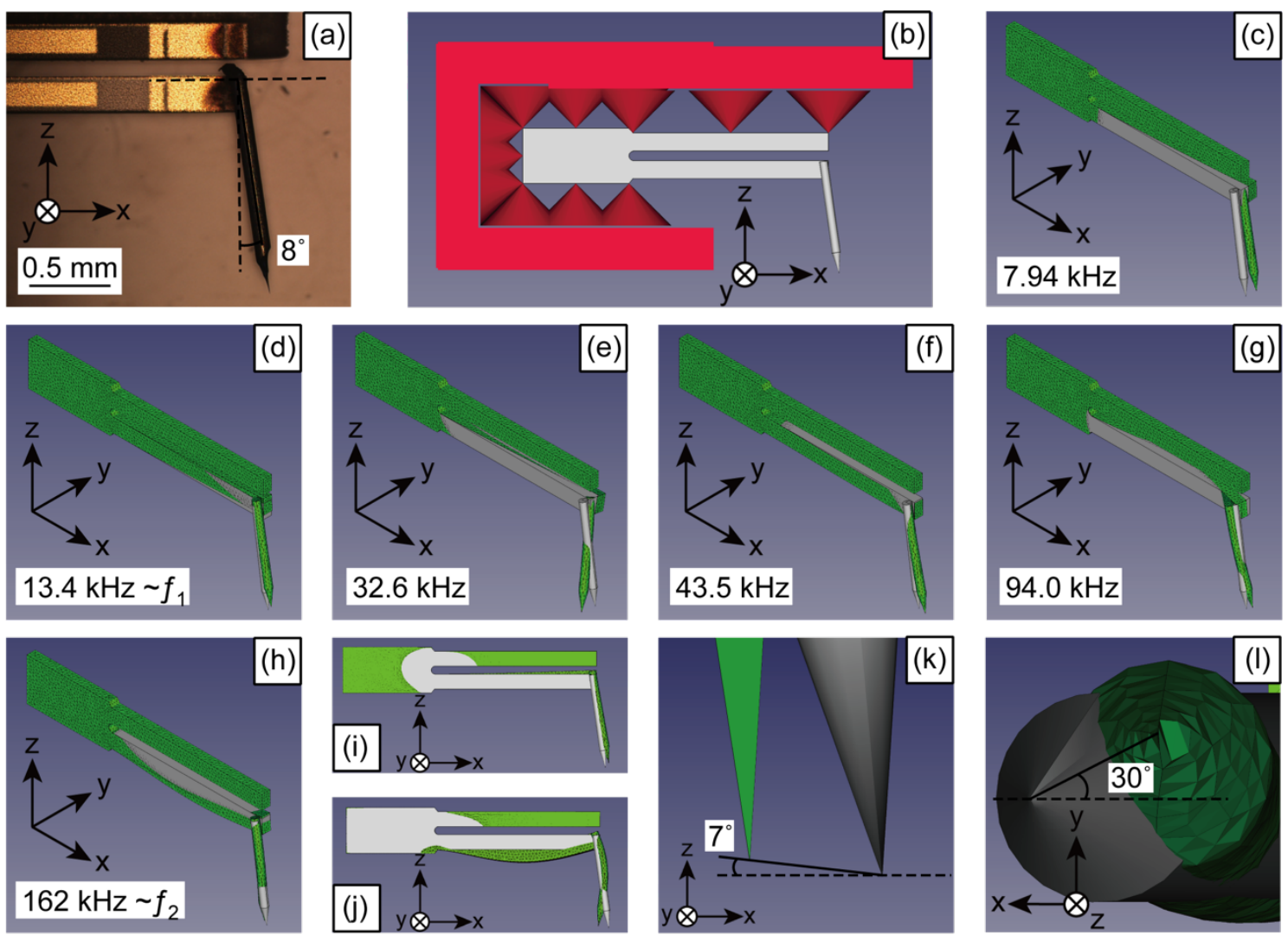

Fig. 5 

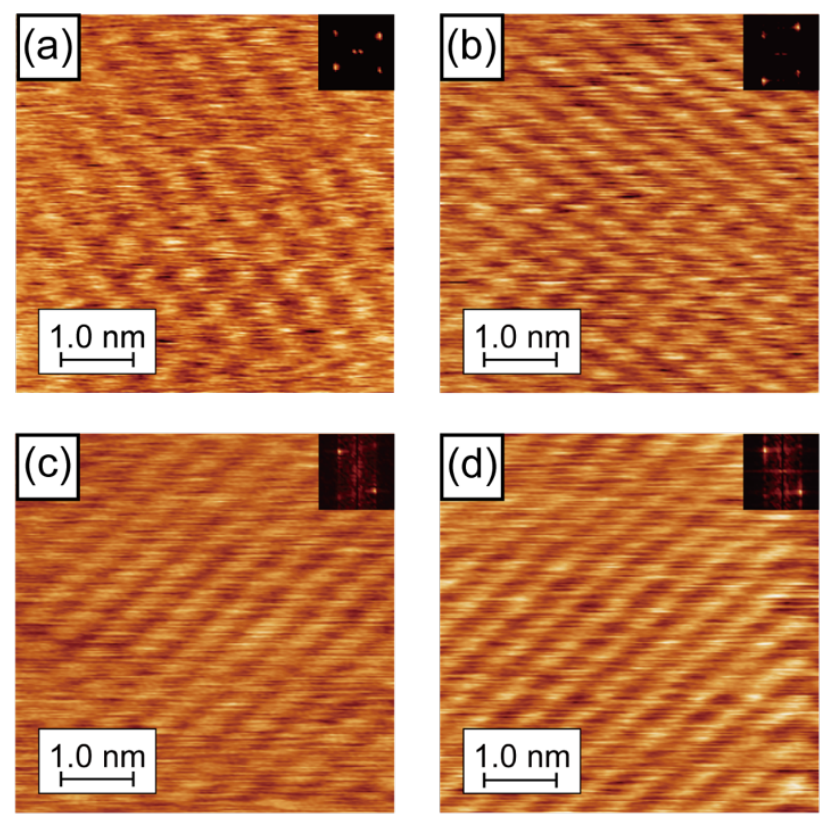

(e)

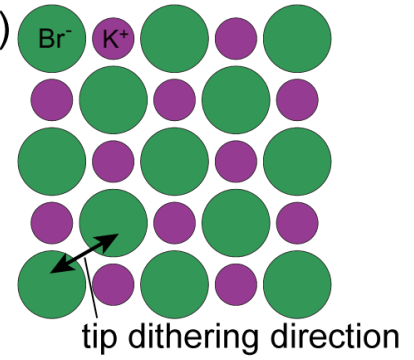

Fig. 6
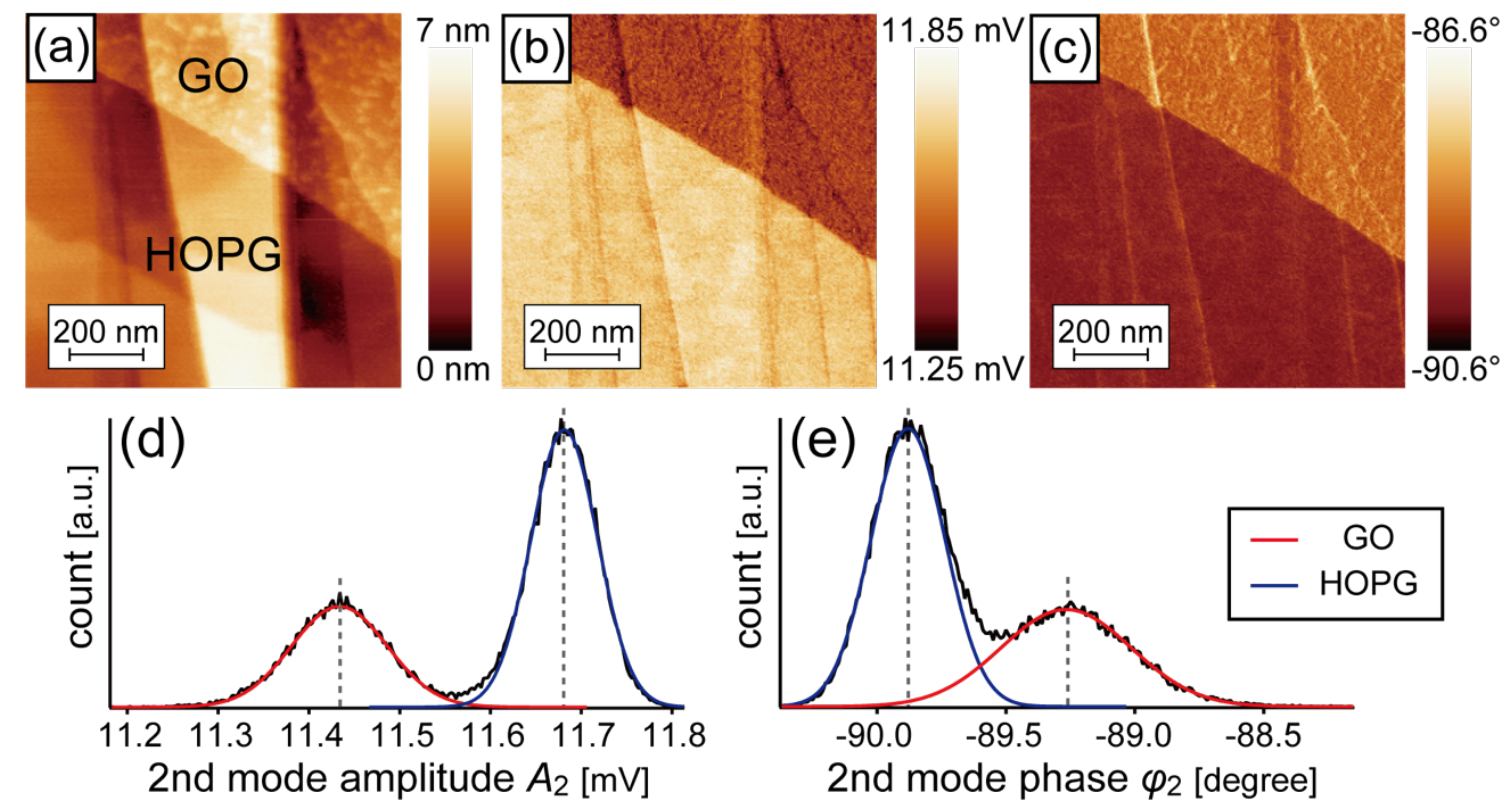

Fig. 7 